International Journal of Environmental Research and

Public Health

ISSN 1660-4601

www.mdpi.com/journal/ijerph

Article

\title{
Effects of Acute Ingestion of Native Banana Starch on Glycemic Response Evaluated by Continuous Glucose Monitoring in Obese and Lean Subjects
}

\author{
Guadalupe Jiménez-Domínguez ${ }^{1}$, Jorge L. Ble-Castillo ${ }^{2, *}$, María A. Aparicio-Trápala ${ }^{3}$, \\ Isela E. Juárez-Rojop ${ }^{2}$, Carlos A. Tovilla-Zárate ${ }^{4}$, Deysi J. Ble-Castillo ${ }^{5}$, \\ Carlos García-Vázquez ${ }^{2}$, Viridiana Olvera-Hernández ${ }^{2}$, Bedelia Pérez-Pimienta ${ }^{6}$, \\ Juan C. Diaz-Zagoya ${ }^{2}$ and José D. Mendez ${ }^{7}$
}

1 Endocrinology Department, General Hospital No. 46, Mexican Institute for Social Security, Villahermosa 86060, Mexico; E-Mail: jimenezg03@hotmail.com

2 Metabolic Diseases Lab, Research Center, Academic Division of Health Sciences, Juarez Autonomous University of Tabasco, Villahermosa 86150, Mexico;

E-Mails: iselajua22@yahoo.com.mx (I.E.J.-R.); gbasecs@hotmail.com (C.G.-V.); viryolvera11@gmail.com (V.O.-H.); zagoya@unam.mx (J.C.D.-Z.)

3 Academic Division of Agricultural Sciences, Juarez Autonomous University of Tabasco, Villahermosa 86280, Mexico; E-Mail: sabina52@hotmail.com

4 Multidisciplinary Academic Division of Comalcalco, Comalcalco 86650, Mexico; E-Mail: alfonsotovillaz@hotmail.com

5 Scool of Medicine, Montemorelos University, Montemorelos 67530 Mexico; E-Mail: deysible@hotmail.com

6 Rodolfo Nieto Padrón Children's Hospital, Secretaria de Salud, Villahermosa 86150, Mexico; E-Mail: bedelia@live.com.mx

7 Medical Research Unit on Metabolic Diseases, Medical Specialities Hospital, Centro Médico Nacional Siglo XXI (CMN-SXXI), Mexican Institute for Social Security, Distrito Federal 06703, Mexico; E-Mail: mendezf@unam.mx

* Author to whom correspondence should be addressed; E-Mail: jblecastillo@hotmail.com; Tel.: +52-993-358-1500 (ext. 6318); Fax: +52-993-351-1132.

Academic Editor: Paul B. Tchounwou

Received: 3 December 2014 / Accepted: 2 February 2015 / Published: 6 July 2015 


\begin{abstract}
An abnormal glycemic profile, including postprandial glycemia and acute glucose spikes, precedes the onset of overt diabetes in obese subjects. Previous studies have shown the beneficial effects of chronic native banana starch (NBS) supplementation. In this study, we examined the effects of acute ingestion of NBS on glycemic profiles by means of continuous glucose monitoring in obese and lean subjects. In a crossover study, obese and lean subjects consumed beverages containing either $38.3 \mathrm{~g}$ of NBS or $38.3 \mathrm{~g}$ of digestible corn starch (DCS) twice daily during 4 days. On day 5, a 3-h meal tolerance test (MTT) was performed to evaluate glucose and insulin responses. After 1 week of washout period, treatments were inverted. NBS supplementation reduced the 48-h glycemia AUC in lean, obese, and in the combined group of lean and obese subjects in comparison with DCS. Postprandial glucose and insulin responses at MTT were reduced after NBS in comparison with DCS in all groups. However, no changes were observed in glycemic variability $(\mathrm{GV})$ indexes between groups. In conclusion, acute NBS supplementation improved postprandial glucose and insulin responses in obese and lean subjects during $48 \mathrm{~h}$ of everyday life and at MTT. Further research to elucidate the mechanism behind these changes is required.
\end{abstract}

Keywords: native banana starch; resistant starch; obesity; glycemic response; continuous glucose monitoring

\title{
1. Introduction
}

Worldwide, the combined prevalence of overweight and obesity rose by $27.5 \%$ for adults and by 47.1\% for children between 1980 and 2013. Mexico is among the countries with the highest prevalence of obesity for both adulthood and childhood [1]. Obesity is one of the major risk factors for Type 2 diabetes (T2DM) and cardiovascular disease (CVD). The critical role of a healthy lifestyle in T2DM prevention has been understood from large-scale intervention studies [2,3]. From these findings, it has been shown that lifestyle changes, including a healthy diet, can reduce the incidence of diabetes in high-risk subjects. It is also known that in addition to postprandial hyperglycemia peaks, glycemic variability $(\mathrm{GV})$, understood as upward and downward acute glucose fluctuations abroad a determined range is associated with oxidative stress damage to the cells [4-7]. These alterations have been linked with the development of diabetes complications and might also be associated with a reduction in time of diabetes onset in obese subjects. Thus, modifications in diet, which can reduce postprandial glycemia excursions or GV, would result in great benefit in diabetes prevention. Previous studies have demonstrated that dietary fibers are associated with improved glycemic control in healthy subjects and in those with diabetes [8]. In this line, resistant starch (RS) is recognized as a dietary fiber that resists digestion in the human small intestine and that reaches partial or complete fermentation in the colon [9]. The short chain fatty acids (SCFA) produced during this process and the increased incret in secretion have been associated with the beneficial effects of dietary fiber on glucose metabolism [10].

Previous studies have demonstrated the beneficial effects of corn resistant starch (Hi-Maize ${ }^{\circledR}$ ) supplementation on glucose metabolism in animal models and in patients with metabolic syndrome or 
with T2DM $[10,11]$. On the other hand, unripe bananas are known to be the non-manufactured food with the highest resistant starch content [12]. Native banana starch (NBS), isolated from the variety Musa (AAA group) Dwarf Cavendish $(F)$ is widely produced in Tabasco and exhibits a high RS content. We have shown that NBS supplementation induced a reduction in fasting glycemia and lipids in diabetic rats as well as in those fed with a high-sucrose diet $[13,14]$. In obese women with diabetes, NBS $24 \mathrm{~g}$ /day for 4 weeks improved insulin sensitivity and reduced body weight [15]. In non-diabetic obese women, NBS $30 \mathrm{~g}$ /day for 8 weeks induced fasting glycemia reduction and increased insulin sensitivity [16].

Interestingly, the recently developed continuous glucose monitoring system (CGMS) provides much more glycemic information, including magnitude, duration, and frequency of blood glucose levels. This tool can be used over several days in patients under free-living conditions, offering more advantages than the simple fasting glycemia or the oral glucose tolerance test (OGTT). In this study, our objective was to determine the effects of acute supplementation of NBS on glycemic profile estimated by 48-h continuous glucose monitoring and during a standardized MTT.

\section{Experimental Section}

\subsection{Study Subjects}

The subjects were recruited from among workers and students of the School of Nutrition of the "Universidad Juarez Autonoma de Tabasco" between January 2013 and December 2013. The experimental protocol was approved by the Ethical Committee of the "Instituto Mexicano del Seguro Social" with number R-2011-2701-50, in compliance with the ethical principles and guidelines for the protection of human subjects under research. The purpose and risks of the study were explained to the participants before they provided written informed consent.

Forty five subjects were screened for obese and lean participants. Anthropometric indexes and basic laboratory examinations were carried out. Participants for the obese group were included if they were healthy persons between 18 and 45 years of age, were obese (World Health Organization [WHO] criterion body mass index $[\mathrm{BMI}]>30 \mathrm{Kg} / \mathrm{m}^{2}$ ), had a normal fasting glycemia (NFG) with values $<100 \mathrm{mg} / \mathrm{dL}(<5.6 \mathrm{mmol} / \mathrm{L}$ ), glycated hemoglobin $(\mathrm{HbAlc})<6.5 \%$ (American Diabetes Association [ADA], 2012), and had maintained stable weight during the three months prior to experimentation. Subjects in the normal-weight groups (lean group) were included if they were between 18 and 45 years of age, had 18.5-24.9 BMI values, had fasting glycemia values $<100 \mathrm{mg} / \mathrm{dL}(<5.6 \mathrm{mmol} / \mathrm{L})$, had $\mathrm{HbA}$ cc values $<6.5 \%$, and had maintained stable weight during the three months prior to experimentation. Subjects not included in this study were those with a previous diagnosis of diabetes, with fasting glycemia $>126 \mathrm{mg} / \mathrm{dL}(>7.0 \mathrm{mmol} / \mathrm{L})$ or glycated hemoglobin $>6.5 \%$ (ADA criteria), with digestive disorders, chronic diseases such as renal or hepatic, being pregnant, under psychiatric treatment, receiving medical or naturist treatment to reduce $\mathrm{BW}$, practicing intense physical activity $(>90$ min per week), receiving immunosuppressants, or with a history of cigarette smoking or alcoholism. Thirty two participants were eligible for the study, 15 for the obese group and 17 for the lean group. However, only 10 subjects in each group completed the whole protocol. 


\subsection{Test Products}

Native banana starch (NBS) was obtained from unripe (green) bananas (Musa [AAA group]) Dwarf Cavendish $(F)$ with a physiological age of 15 weeks obtained from a fruit packing plant located at Km. 43.5 of the Villahermosa-Teapa highway in the Mexican state of Tabasco. The NBS was isolated by means of a previously described procedure [17]. Briefly, after washing, the bananas were peeled, cut into $5-6 \mathrm{~cm}^{3}$ pieces, immediately rinsed in citric acid solution, and then macerated at low speed in an industrial blender for $2 \mathrm{~min}$. The homogenate was consecutively sieved through screens (30, 80, and 100-U.S. mesh) and washed with distilled water; it was then centrifuged at 10,000 rpm. The sediment was further purified by washing and centrifugation. The white starch sediment was dried in a convection oven at $40-45^{\circ} \mathrm{C}$, passed through a 100-mesh screen, and stored at room temperature in sealed glass jars. Proximate analysis included the following: 3.38\% of moisture content; $1.88 \%$ protein; $0.4281 \%$ fat, and $0.78 \%$ ash $(14.003,14.057,14.059$, and 14.006, AOAC international recommended methods). Water activity $\left(a_{w}\right)$ of NBS was measured using AquaLab equipment and resulted in 0.59 . Resistant starch (RS) content measured according to the Goñi et al. method was found to be $34 \%$ on a dry weight basis [18]. Digestible corn starch (DCS) was purchased from Unilever de México, S. de R.L. de C.V. as Maizena ${ }^{\circledR}$ containing $94.4 \%$ rapid digestible starch.

\subsection{Study Design and Protocol}

A randomized, crossover, single-blind controlled trial with two 5-day treatment periods and a 7-day washout was conducted. The study protocol for all participants in a 5-day treatment period is shown in Figure 1. On day 1, subjects were trained to use the monitor system and perform the calibrations. On day 2, the CGM system sensor was inserted. From days 1-4 of the treatment period, subjects were under a low-fiber diet, consumed two beverages daily, and were requested to perform daily food recording. On day 5, a 3-h MTT was carried out and the CGMS sensor was removed. After 5 days of supplementation, a 7-day washout period was performed. Supplementation assignments were then reversed for an additional 5 days of follow-up.

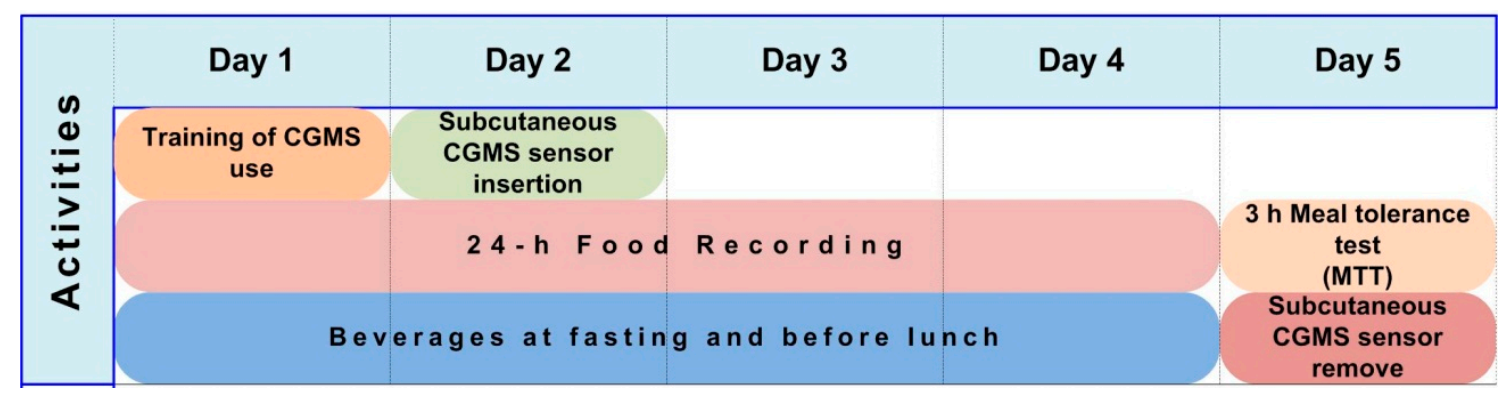

Figure 1. Study Protocol for all participants.

\subsection{Treatments}

All of the subjects received low-fiber diets at breakfast and lunch from the Nutrition Department's restaurant during days $1-4$ of the treatment period. The low-fiber diet consisted of avoiding whole-grain/wholemeal breads and cereals, legumes, nuts, and seeds, and of removing the 
skins from fruit and vegetables. Subjects were requested to maintain this diet at dinner and to perform daily food recording before they went to bed. Subjects were randomized to receive two beverages daily containing either $38.39 \mathrm{~g}$ of NBS or $38.39 \mathrm{~g}$ of DCS during 4 days of the treatment period. The NBS beverage contained $38.39 \mathrm{~g}$ of NBS, $31.82 \mathrm{~g}$ of soy milk, and $240 \mathrm{~mL}$ of purified water. The DCS beverage contained $38.39 \mathrm{~g}$ of DCS, $31.82 \mathrm{~g}$ of soy milk, and $240 \mathrm{~mL}$ of purified water. Beverages were consumed in the fasting state (7:00 A.M.-9:00 A.M.) and before lunch (1:00 P.M.-3:00 P.M.). Individuals in the NBS group consumed $26.7 \mathrm{~g}$ /day of banana-resistant starch during the first four days of the treatment period. Beverages were prepared and given to the subjects at the restaurant. The doses of NBS used in the present study were based on previous studies from other authors and from our group. Robertson et al. administered Hi-Maize ${ }^{\circledR} 50 \mathrm{~g} /$ day containing $30 \mathrm{~g}$ of RS during four weeks to healthy subjects [19] or Hi-Maize ${ }^{\circledR} 67 \mathrm{~g}$ containing $40 \mathrm{~g}$ of RS daily during 12 weeks to patients with T2DM [10]. In previous studies, we employed NBS $30 \mathrm{~g} /$ day for eight weeks in obese women without diabetes with good tolerability [16]. Compliance was assessed by counting unopened sachets and by a query regarding the missed servings.

\subsection{Continuous Glucose Monitoring}

All subjects were monitored by the continuous glucose monitoring system (CGMS; Guardian ${ }^{\circledR}$ REAL-Time CGMS; Medtronic, Northridge, CA, USA) for $72 \mathrm{~h}$. On day 1, participants were instructed on how to use the monitoring system and perform, by themselves, at least three calibration readings per day using the capillary blood sampling method (OneTouch ${ }^{\circledR}$ Ultra $^{\circledR} 2$ from Johnson \& Johnson, New Brunswick, NJ, USA). All subjects were instructed to avoid strenuous exercise during the experimental period. The CGM system sensor microelectrode was inserted into subcutaneous (s.c.) tissue in the subjects' periumbilical region at the clinical site on day 2 and removed on day 5. Criteria for optimal accuracy of the CGM were satisfied [20]. To investigate GV, analysis was limited to data obtained from the intermediate 48-h recording (days 3 and 4 ) to avoid bias due to insertion and removal of the CGMS sensor. The recorded data were downloaded into a personal computer for analysis of glucose excursions parameters.

\subsection{Assessment of Glycemic Variability $(G V)$}

Standard deviation (SD) of blood glucose, coefficient of variation (CV), mean amplitude of glycemic excursions (MAGE), and mean absolute glucose (MAG) changes were used to assess GV. The SD was obtained through data management with GraphPad Prism software (La Jolla, CA, USA). MAGE, considered a gold standard of GV, was calculated by measuring the arithmetic mean of the differences between consecutive peaks and nadirs, provided that the differences were $>1$ SD of the mean glucose value. MAG change takes into account all glycemic variations over time, including those remaining within the physiological range. MAG-5 was obtained by calculating absolute increments and decrements of glucose from peaks to nadirs for $5 \mathrm{~min}$ according to the formula $\mathrm{MAG}=\Delta \mathrm{Glucose} / \Delta \mathrm{Time}$ as described by Hermanides et al. [21]. 


\subsection{Meal Tolerance Test}

A standardized MTT was conducted on day 5 in subjects at fasting state. On arrival at the Investigation Unit, an indwelling catheter was inserted into an antecubital vein in the forearm of the subject for withdrawal of blood samples. Five min after catheter placement, the first blood sample was taken $(t=0)$. Then, the participants were offered their respective beverage and a Kellogg's ${ }^{\circledR}$ Rice Krispies ${ }^{\circledR}$ bar (Kellogg's Company, Battle Creek, MI, USA), which had to be consumed at least within 5 min. A 22-g bar of Kellogg's ${ }^{\circledR}$ Rice Krispies ${ }^{\circledR}$ contains total fat 2 g, total carbohydrates 17 g, protein $<1 \mathrm{~g}$, sodium $105 \mathrm{mg}$, and dietary fiber, 0 . Total calories from the bar were $90 \mathrm{kcal}$. Considering the nutritional content of the beverages, each subject consumed $391 \mathrm{kcal}$ in this test. Additional blood samples were drawn at 30,60, 90, 120, 150, and $180 \mathrm{~min}$ for glucose and insulin determinations. Subjects remained at the Investigation Unit under observation during the test period and were allowed to read, watch television, or talk.

\subsection{Biochemical Measurements}

Blood samples were collected by trained personnel and serum samples were immediately frozen and stored at $-70{ }^{\circ} \mathrm{C}$ until biochemical determination. Glucose, cholesterol, triglycerides, and insulin concentrations were performed using the Architect Clinical Chemistry Autoanalyzer System from Abbott Laboratories (Chicago, IL, USA). Glucose concentration was determined by enzymatic assay and insulin was measured using chemiluminiscent microparticle immunoassay (CMIA). The total coefficient of variation for the glucose and insulin assays were $5 \%$ and $<7 \%$, respectively. Insulin resistance was estimated according to Homeostasis Model Assessment (HOMA-IR), which was calculated by means of the product of fasting plasma glucose $(\mathrm{mmol} / \mathrm{L})$ and insulin $(\mu \mathrm{U} / \mathrm{mL})$ divided by 22.5 [22].

\subsection{Statistical Analysis}

Data are expressed as means \pm standard error of the mean (SEM) values, unless otherwise specified. Given the crossover design of the study, endpoint analyses were carried out for completers only. The D'Agostino-Pearson normality test was performed to assess whether the data were consistent with Gaussian distribution. Total area under the curve (AUC) was calculated using the trapezoid method. Two-tailed paired Student $t$ test was employed for comparisons between outcomes from the same subjects with different treatments, or with the unpaired Student $t$ test for comparisons between obese and lean subjects. Time course data were also analyzed by repeated measures analysis of variance (ANOVA) for comparison. Differences were considered as statistically significant for $p$ values $<0.05$. Data were processed and analysed using GraphPad Prism ver. 6.00 statistical software (GraphPad Software, Inc., La Jolla, CA, USA). 


\section{Results and Discussion}

\subsection{Characteristics of Subjects}

From the lean group, seven subjects were excluded, six for technical problems and one for personal reasons. From the obese subjects, three exhibited technical problems and two withdrew informed consent. The main technical problems included the sensor and monitor use and difficulties in performing the recommended daily calibrations. Baseline characteristics at screening of the 20 participants who completed the treatments are displayed in Table 1 . The majority of the participants were young women near the age of 30 years. As expected, obese subjects exhibited higher BMI values compared with lean subjects. All of these were classified as type I obese, and $60 \%$ exhibited abdominal adiposity according to WHO criteria. All of the participants exhibited fasting normoglycemia according to the ADA, with glycemia values $<100 \mathrm{mg} / \mathrm{dL}$ and HbAlc levels $<6.5 \%$. According to the crossover design utilized in this study, each subject received both treatments, NBS and DCS, in a random assignation process.

Table 1. Baseline characteristics of the study subjects.

\begin{tabular}{cccc}
\hline Characteristic & Lean & Obese & $\boldsymbol{p}$ \\
\hline$N$ of subjects & 10 & 10 & -- \\
Gender $(\mathrm{M} / \mathrm{F})$ & $1 / 9$ & $1 / 9$ & - \\
Age $($ years $)$ & $26.4 \pm 7.86$ & $31.6 \pm 4.97$ & 0.094 \\
BMI $\left(\mathrm{kg} / \mathrm{m}^{2}\right)$ & $23.27 \pm 1.22$ & $30.73 \pm 1.07$ & $<0.0001$ \\
Glucose $(\mathrm{mmol} / \mathrm{L})$ & $4.25 \pm 0.57$ & $4.60 \pm 0.31$ & 0.105 \\
HbAlc $(\%)$ & $5.18 \pm 0.51$ & $5.03 \pm 0.58$ & 0.547 \\
Total cholesterol $(\mathrm{mmol} / \mathrm{L})$ & $4.48 \pm 0.92$ & $4.99 \pm 0.75$ & 0.192 \\
HDL-Cholesterol $(\mathrm{mmol} / \mathrm{L})$ & $1.27 \pm 0.26$ & $1.23 \pm 0.07$ & 0.629 \\
Triglycerides $(\mathrm{mmol} / \mathrm{L})$ & $1.36 \pm 0.84$ & $1.50 \pm 0.18$ & 0.620 \\
\hline
\end{tabular}

All values are expressed as mean \pm SD. Blood samples were obtained at fasting state. An unpaired Student $t$ test was performed. BMI $=$ Body mass index.

\subsection{Tolerability and Adverse Effects}

Mean daily macronutrient intake from 4-day diet diaries did not differ between treatments. Although there was no need to interrupt the experiments, three of ten subjects treated with NBS informed gastrointestinal discomfort manifested as bloating, flatulence, and abdominal cramping. In the DCS group, two of ten participants reported these adverse effects.

\subsection{Glycemic Excursions in 48-h CGM}

The effects of NBS vs. DCS on glycemic characteristics in 48-h CGM are illustrated in Table 2. Mean blood glucose (MBG) and the total glucose AUC-48 h were both significantly reduced after NBS supplementation in comparison with DCS in all of the groups $(p<0.0001$ for MBG, and $p<0.05$ for AUC-48-h). No statistical differences were observed between NBS and DCS supplementation on GV indexes, including SD, CV, MAGE, and MAG5 changes between groups. 
Table 2. Effects of beverages containing native banana starch (NBS) or digestible corn starch (DCS) in glycemic characteristics based on continuous glucose monitoring (CGM).

\begin{tabular}{|c|c|c|c|c|c|c|c|c|c|}
\hline \multirow{2}{*}{ Characteristic } & \multicolumn{3}{|c|}{ Lean } & \multicolumn{3}{|c|}{ Obese } & \multicolumn{3}{|c|}{ Lean and Obese } \\
\hline & DCS & NBS & $p$ & DCS & NBS & $p$ & DCS & NBS & $p$ \\
\hline MBG $(\mathrm{mmol} / \mathrm{L})$ & $5.83 \pm 0.95$ & $5.15 \pm 0.71$ & $<0.0001$ & $6.28 \pm 0.82$ & $5.44 \pm 0.63$ & $<0.0001$ & $6.06 \pm 0.89$ & $5.29 \pm 0.67$ & $<0.0001$ \\
\hline AUC (mmol.h/L) & $4.43 \pm 0.49$ & $3.97 \pm 0.45$ & 0.0329 & $5.85 \pm 0.29$ & $5.06 \pm 0.20$ & 0.0121 & $5.16 \pm 0.32$ & $4.51 \pm 0.27$ & 0.0007 \\
\hline $\mathrm{SD}(\mathrm{mmol} / \mathrm{L})$ & $1.00 \pm 0.44$ & $0.77 \pm 0.25$ & 0.1229 & $1.13 \pm 0.39$ & $1.07 \pm 0.46$ & 0.7651 & $1.07 \pm 0.41$ & $0.92 \pm 0.40$ & 0.2440 \\
\hline CV (\%) & $16.81 \pm 5.88$ & $15.14 \pm 4.88$ & 0.3904 & $17.74 \pm 4.60$ & $20.25 \pm 9.64$ & 0.5028 & $17.28 \pm 5.16$ & $17.70 \pm 7.88$ & 0.8374 \\
\hline $\operatorname{MAGE}(\mathrm{mmol} / \mathrm{L})$ & $2.32 \pm 0.67$ & $2.26 \pm 0.76$ & 0.8780 & $2.27 \pm 1.07$ & $1.77 \pm 0.61$ & 0.2100 & $2.30 \pm 0.87$ & $2.02 \pm 0.72$ & 0.2960 \\
\hline $\mathrm{MAG}_{5}(\mathrm{mmol} / \mathrm{L})$ & $0.12 \pm 0.04$ & $0.10 \pm 0.02$ & 0.2176 & $0.12 \pm 0.03$ & $0.15 \pm 0.08$ & 0.3095 & $0.12 \pm 0.04$ & $0.13 \pm 0.07$ & 0.7622 \\
\hline
\end{tabular}

Data represent Mean $\pm \mathrm{SD}$. AUC $=$ Area under the curve; $\mathrm{MBG}=$ Mean blood glucose; $\mathrm{SD}=$ Standard deviation; $\mathrm{CV}=\mathrm{Coefficient}$ of variation; $\mathrm{MAGE}=\mathrm{Mean}$ amplitude

of glycemic excursions, MAG5 = Mean absolute glucose changes for $5 \mathrm{~min}$. A paired Student $t$ test was used for comparisons. 
When comparing obese and lean subjects, the obese group exhibited a far greater AUC-48-h in comparison with lean subjects in both NBS and DCS supplementation $(p=0.034$ and $p=0.026$, respectively). However, no changes were observed in any of the GV indexes between obese and lean groups.

\subsection{Excursions Exceeding Oral Glucose Tolerance Test (OGTT) Thresholds}

Other findings showed that in the lean group, four participants experienced excursions into the impaired glucose tolerance (IGT) range $>7.77 \mathrm{mmol} / \mathrm{L}(>140 \mathrm{mg} / \mathrm{dL}$ ) during a period of $>2.0 \mathrm{~h}$. In the obese group, two subjects exhibited excursions $>11.10 \mathrm{mmol} / \mathrm{L}(>200 \mathrm{mg} / \mathrm{dL})$ during $>1 \mathrm{~h}$ and four subjects, excursions of $>7.77 \mathrm{mmol} / \mathrm{L}$ during a period of $>2.0 \mathrm{~h}$.

\subsection{Meal Tolerance Test (MTT)}

To better control postprandial glucose and insulin responses a standardized MTT was conducted on day 5. A reduction in glycemic and insulin responses after NBS was observed in all of the studied groups. Total glucose AUC-180 min and total insulin AUC-180 min were both reduced following NBS compared to DCS group (Figure 2). When comparing obese and lean subjects (Figure 3), no differences were appreciated in glucose AUC-180 min or insulin AUC-180 min after DCS. Likewise, there was not significant difference in the glycemic response between groups after NBS. However, insulin response (AUC-180 min) tended to be significantly decreased in lean subjects following NBS $(p=0.051)$.
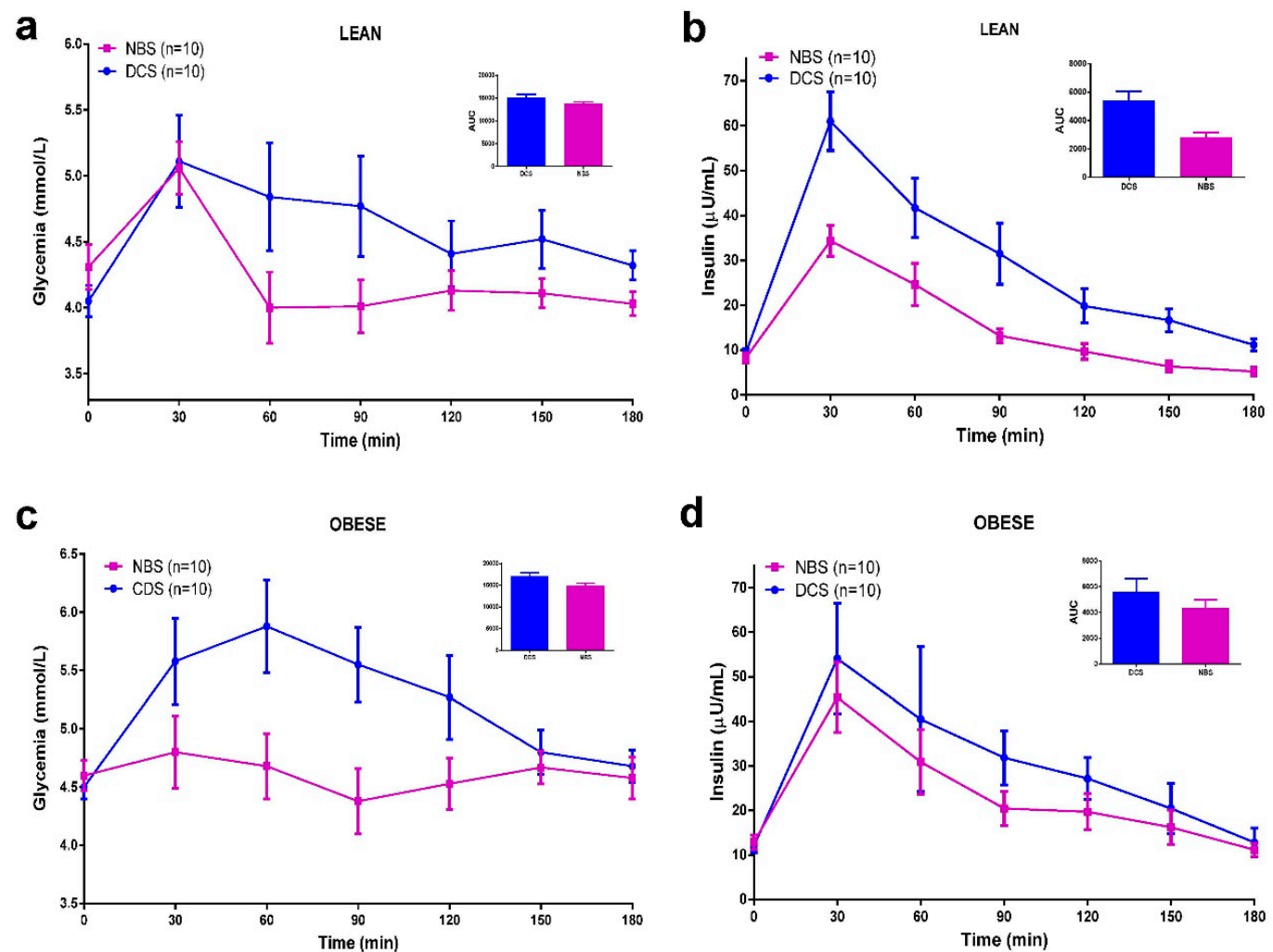

d

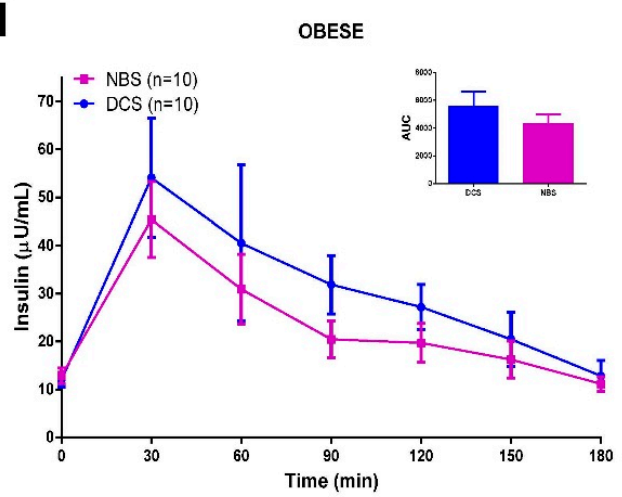

Figure 2. Cont. 

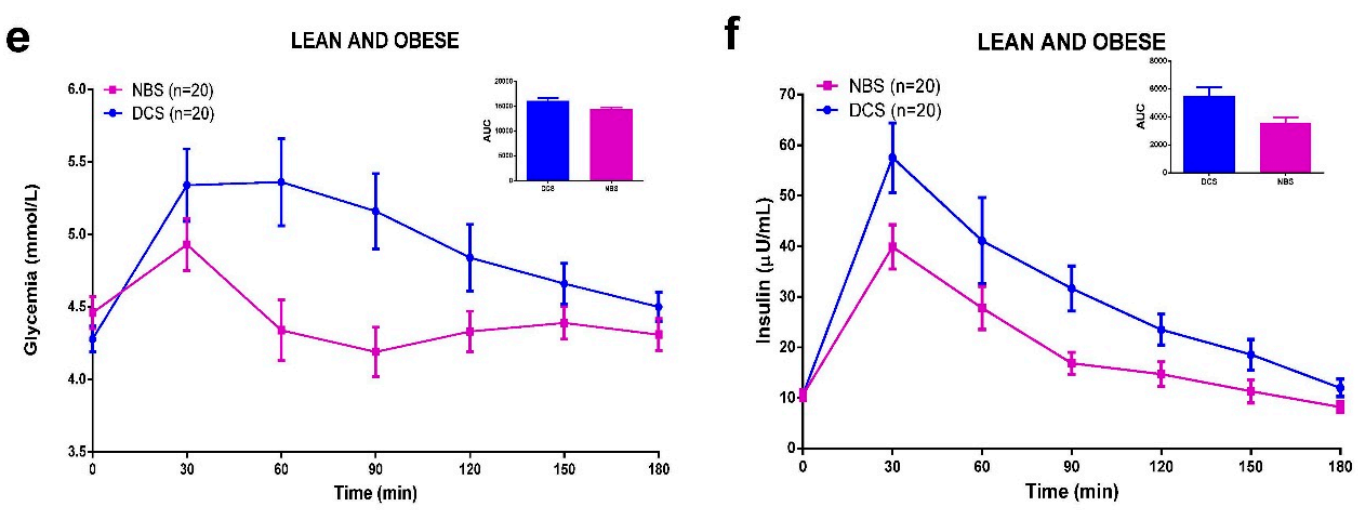

Figure 2. Effects of beverages containing native banana starch (NBS) (red) in comparison with digestible corn starch (DCS) (blue) at the Meal Tolerance Test (MTT) (a,b) in lean group; (c,d) in obese group; $(\mathbf{e , f )}$ in lean and obese group. Notes: Beverages were administered $5 \mathrm{~min}$ before the standardized food. Areas under the curve (AUC) were compared using a paired Student $t$ test or Wilcoxon test. In (a) ANB $v s$. DCS $p=0.0098$; in (b) $p=0.0018$; in (c) $p=0.0223$; in (d) $p=0.0394$; in (e) $p<0.0001$; in (f) $p=0.0002$.

a

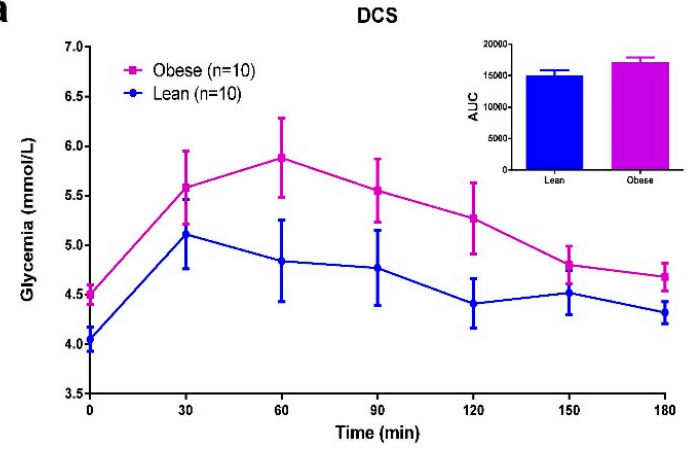

C

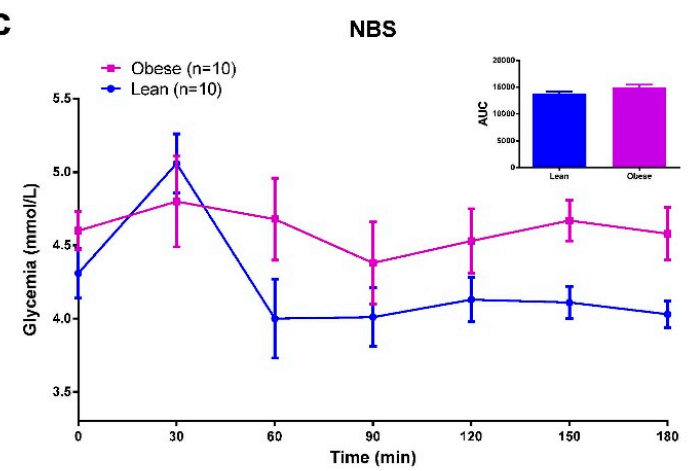

b

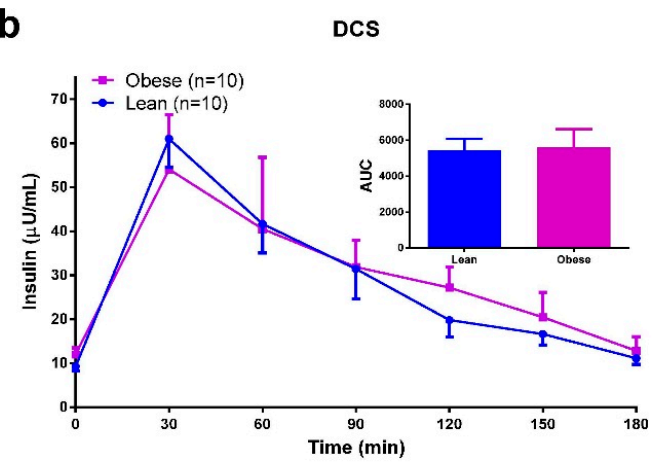

d

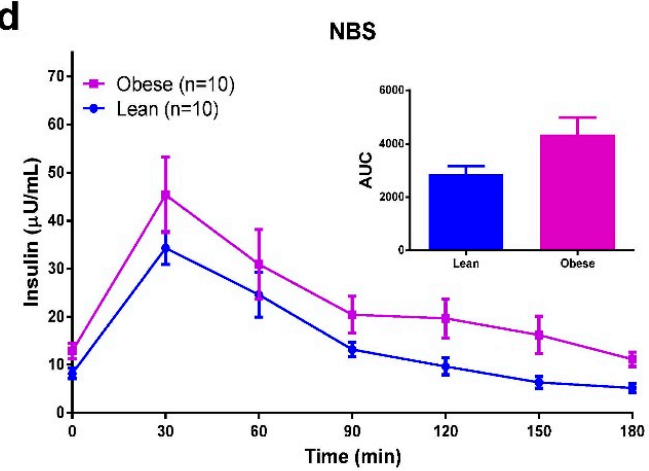

Figure 3. Differences between lean and obese subjects in glycemic and insulin responses during a Meal Tolerance Test (MTT) (a,b) Beverage containing digestible corn starch (DCS). (c,d) Beverage containing native banana starch (NBS). Notes: Beverages were administered $5 \mathrm{~min}$ before the standardized food. Areas under the curve (AUC) were compared using an unpaired Student $t$ test. In (a) Obese $v s$. Lean $p=0.0853$; in (b) $p=0.8892$; in (c) $p=0.0749$; in (d) $p=0.051$. 


\subsection{Discussion}

We showed that acute supplementation of NBS significantly reduces glucose excursions measured by 48-h CGM and also diminished glycemic and insulin responses during a MTT in both lean and obese subjects. However, no changes on GV indexes were observed between groups. In comparison with previous studies, the experimental design of this work is novel in that it employed CGMS to estimate glucose excursions and GV over several days in subjects under conditions similar to those of everyday life.

All of the participants were included with normal fasting glycemia and normal HbA1c values, however, we could observe that some participants were undergoing excursions into glucose intolerant and/or diabetes range during the 48-h CGM. These results confirm previous observations that dysglycemia may be underestimated by traditional methods such as fasting glycemia and HbAlc, and that CGM could serve as a better tool to detect early alterations [23-25]. Likewise, this study also confirmed altered glucose management in obese subjects with normoglycemia and normal HbA1c. Obese subjects exhibited a far greater glycemic burden in comparison with lean subjects regardless of the supplement employed. This disturbance is based on the fact that obese subjects exhibit an early decline in beta cell function, predisposing to an insulin resistance status several years prior to the onset of diabetes.

In both lean and obese subjects, NBS supplementation induced a significant reduction in 48-h glycemic excursions in comparison with DCS. However, in order to better control the postprandial response, an MTT was performed on day 5. In contrast with OGTT, which utilized a pure glucose load, the mixed meal has the advantage of mimicking the "real life" condition. In this test, we observed that NBS significantly reduced glycemic and insulinic postprandial responses in obese and lean subjects. This finding is very important because a recent study in overweight/obese subjects demonstrated that postprandial glycemia is the main contributor to overall 24-h hyperglycemia. Moreover, these authors reported that the contribution is higher when HbAlc values are low, as is the case of many obese subjects with unknown dysglycemia, such as those included in the present study [26].

This effect of NBS in controlling postprandial glycemia under real-life conditions provides great interest with regard to clinical prevention, because it is known that high postprandial glycemia levels occur prior to the deterioration of fasting glucose levels and diabetes onset [27]. Moreover, postprandial hyperglycemia has greater consequences than increased fasting glycemia. In a previous report, increased mortality was observed in subjects with abnormal 2-h postprandial glycemia, but not in those with increased fasting glycemia [28]. In general, it is accepted that hyperglycemic peaks and hyperinsulinemia increases the risk for cardiovascular disease (CVD) in patients with prediabetes $[29,30]$. A reduction in postprandial responses over long periods could be a preventive factor for reducing the onset of diabetes in high-risk subjects and could diminish the incidence of cardiovascular events in individuals with T2DM [31]. The dietary intervention using products manufactured with NBS might be implemented in order to prevent diabetes and improve cardiovascular prognosis.

Despite altered postprandial glucose excursions, no increased GV indices were observed in the obese subjects group and no effect of NBS on GV was detected. Some of the GV index values, such as SD, CV, and MAGE from this group, were similar to those reported in healthy Chinese population measured in 434 individuals 20-60 years old [32]. The lack of alterations in GV indexes could be partially explained by the 
relatively youth of the subjects and because only 6 of 10 exhibited a moderated abdominal obesity. Indeed, few studies have investigated GV in normoglycemic populations. Ma Chung-Ming et al. in 2011 [33] reported increased GV in abdominally obese subjects with normal glucose tolerance (NGT). However, this study was performed in males, and our study included predominantly women.

Diminished glucose and insulin response after NBS could be partially explained by the reduced rate of digestion [12,34]. However, it could also be attributed to increased short chain fatty acids (SCFA) after RS fermentation in the colon. In previous studies, we demonstrated increased SCFA production in rodent intestine after NBS [14]. Colonic SCFA production has been linked with increased secretion of incretin hormones, such as GLP-1 and PYY, from enteroendocrine cells [19,35]. Plasma SCFA have also shown to inhibit adipose tissue lipolysis in vivo: thus, RS might modulate insulin sensitivity through alterations in this fatty acid flux [10,11]. In a recent study in humans, Robertson et al. [36] suggested that the most important effect of chronic supplementation with Hi-Maize ${ }^{\circledR}$ was due to an improvement of the adipose tissue physiological function. As a secondary effect, increased glucose uptake into skeletal muscle was followed. The authors found that Hi-Maize ${ }^{\circledR}$ 8-week supplementation induced stimulation of the expression of lipoprotein-lipase (LPL), adipose triglyceride lipase (ATGL), and hormone-sensitive lipase (HSL) in adipose tissue. From these observations, it is clear that further mechanistic studies are required to investigate the effects of NBS.

Some limitations merit consideration. First, we did not perform an OGTT to rule out glycemic alterations such as diabetes and IGT. Second, this study was conducted predominantly in females because in Mexico, diabetes prevalence is higher in women; thus, the results cannot be extrapolated to male population. Third, we matched the supplements only for total carbohydrates. The advantages of our study, however, comprised the use of a reliable CGMS to investigate glycemic profiles under conditions similar to those of everyday life and the crossover design, which permitted reducing inter-subject variability.

\section{Conclusions}

In this study, we demonstrated that acute supplementation of NBS improved 48-h glycemic excursions and postprandial glucose and insulin responses in obese and lean subjects. However, no differences in glycemic variability indexes were found between subjects and treatments. These results are relevant because NBS supplementation could represent an inexpensive and accessible alternative to contribute to diabetes and cardiovascular disease prevention in high-risk patients.

\section{Acknowledgments}

This study was part of the dissertation of Guadalupe Jiménez-Domínguez. We are grateful to Rubén Córdova-Uscanga, for his clinical laboratory assistance, to Saraí Aguilar Barojas for her valuable help in subjects screening. We would particularly like to thank to the volunteers who made this study possible. This study was supported by the "Universidad Juárez Autónoma de Tabasco" (UJAT), Grant OP/PIFI-2013-27MSV0018V-07-01 and the "Programa de Fomento a la Investigación" (UJAT-2012-1A-45). 


\section{Author Contributions}

Guadalupe Jimenez-Dominguez carried out the clinical protocol including Continuous Glucose Monitoring System (CGMS) performance and subject supervision. Jorge L. Ble-Castillo conceived of the idea, organized the contents, supervised data collection, and prepared the manuscript. Maria A. Aparicio-Trápala supervised NBS production and drafted the manuscript. Isela E. Juárez-Rojop organized some of the experimental sessions and drafted the manuscript. Carlos A. Tovilla-Zárate aided in the statistical analysis and drafted the manuscript. Deysi J. Ble-Castillo helped with the analysis and drafted the manuscript. Carlos García-Vázquez helped with some clinical determinations, the statistical analysis and formatting the manuscript. Viridiana Olvera-Hernández helped supervising Native Banana Starch (NBS). production and results analysis. Bedelia Pérez-Pimienta aided in performing the Meal Tolerance Test (MTT) and drafting. Juan C. Diaz-Zagoya participated in the study design, statistical analysis and drafted the manuscript. Jose D. Méndez helped with the statistical analysis and drafted the manuscript. All authors read and approved the final manuscript.

\section{Conflicts of Interest}

The authors declare no conflict of interest.

\section{References}

1. Ng, M.; Fleming, T.; Robinson, M.; Thomson, B.; Graetz, N.; Margono, C.; Mullany, E.C.; Biryukov, S.; Abbafati, C.; Abera, S.F.; et al. Global, regional, and national prevalence of overweight and obesity in children and adults during 1980-2013: A systematic analysis for the Global Burden of Disease Study 2013. Lancet 2014, 384, 766-781.

2. Tuomilehto, J.; Lindström, J.; Eriksson, J.G.; Valle, T.T.; Hämäläinen, H.; Ilanne-Parikka, P.; Keinänen-Kiukaanniemi, S.; Laakso, M.; Louheranta, A.; Rastas, M.; et al. Prevention of type 2 diabetes mellitus by changes in lifestyle among subjects with impaired glucose tolerance. N. Engl. J. Med. 2001, 344, 1343-1350.

3. Knowler, W.C.; Barrett-Connor, E.; Fowler, S.E.; Hamman, R.F.; Lachin, J.M.; Walker, E.A.; Nathan, D.M.; Diabetes Prevention Program Research Croup. Reduction in the incidence of type 2 diabetes with lifestyle intervention or metformin. N. Engl. J. Med. 2002, 346, 393-403.

4. Brownlee, M. The pathobiology of diabetic complications: A unifying mechanism. Diabetes 2005, 54, 1615-1625.

5. Ceriello, A. Postprandial Hyperglycemia and cardiovascular disease: Is the HEART2D study the answer? Diabetes Care 2009, 32, 521-522.

6. Nathan, D.M. Navigating the choices for diabetes prevention. N. Engl. J. Med. 2010, 362, 1533-1535.

7. Monnier, L.; Mas, E.; Ginet, C.; Michel, F.; Villon, L.; Cristol, J.P.; Colette, C. Activation of oxidative stress by acute glucose fluctuations compared with sustained chronic hyperglycemia in patients with type 2 diabetes. JAMA 2006, 295, 1681-1687.

8. Post, R.E.; Mainous, A.G.; King, D.E.; Simpson, K.N. Dietary fiber for the treatment of type 2 diabetes mellitus: A meta-analysis. J. Am. Board Fam. Med. 2012, 25, 16-23. 
9. Jones, J. CODEX-aligned dietary fiber definitions help to bridge the "fiber gap". Nutr. J. 2014, 13, doi:10.1186/1475-2891-13-34.

10. Bodinham, C.L.; Smith, L.; Thomas, E.L.; Bell, J.D.; Swann, J.R.; Costabile, A.; Russell-Jones, D.; Umpleby, A.M.; Robertson, M.D. Efficacy of increased resistant starch consumption in human type 2 diabetes. Endocr. Connect. 2014, 3, 75-84.

11. Robertson, M.D. Dietary-resistant starch and glucose metabolism. Curr. Opin. Clin. Nutr. Metab. Care 2012, 15, 362-367.

12. Fuentes-Zaragoza, E.; Riquelme-Navarrete, M.J.; Sánchez-Zapata, E.; Pérez-Álvarez, J.A. Resistant starch as functional ingredient: A review. Food Res. Int. 2010, 43, 931-942.

13. Pérez-Sánchez, E. Efectos del Consumo de una Formulación que Incluye Almidón Resistente de Plátano en la Glucemia Postprandial de Pacientes con Diabetes Mellitus Tipo 2. Tesis de Maestría en Ciencias Alimentarias; Universidad Juárez Autónoma de Tabasco: Villahermosa, Tabasco, México, 2007.

14. Olvera-Hernández, V. Almidón Resistente de Banano Enano Gigante (Cavendish AAA) en la Glicemia y Producción de Ácidos Grasos de Cadena Corta en Ratas con Síndrome Metabólico. Tesis de Maestría en Ciencias Alimentarias; Universidad Juárez Autónoma de Tabasco: Villahermosa, Tabasco, México, 2010.

15. Ble-Castillo, J.L.; Aparicio-Trápala, M.A.; Francisco-Luria, M.U.; Córdova-Uscanga, R.; Rodríguez-Hernández, A.; Méndez, J.D.; Díaz-Zagoya, J.C. Effects of Native Banana Starch Supplementation on Body Weight and Insulin Sensitivity in Obese Type 2 Diabetics. Int. J. Environ. Res. Public Health 2010, 7, 1953-1962.

16. Ble Castillo, J.; Aparicio Trápala, M.; Gómez Vázquez, A.; Rodríguez Hernández, A.; Mendez, J.; Juárez Rojop, I.; Aguilar Mariscal, H.; Ramón Frías, T.; Díaz Zagoya, J. Potential beneficial effects of native banana starch on glycemia and insulin resistance in obese nondiabetic women. Interciencia 2012, 37, 470-476.

17. Flores-Gorosquera, E.; Garcia-Suarez, F.J.; Flores-Huicochea, E.; Nunez-Santiago, M.C.; Gonzalez-Soto, R.A.; Bello-Perez, L.A. Yield of starch extraction from plantain (Musa paradisiaca). Pilot plant study. Acta Cient. Venez. 2004, 55, 86-90.

18. Goñi, I.; García-Diz, L.; Mañas, E.; Saura-Calixto, F. Analysis of resistant starch: A method for foods and food products. Food Chem. 1996, 56, 445-449.

19. Robertson, M.D.; Bickerton, A.S.; Dennis, A.L.; Vidal, H.; Frayn, K.N. Insulin-sensitizing effects of dietary resistant starch and effects on skeletal muscle and adipose tissue metabolism. Am. J. Clin. Nutr. 2005, 82, 559-567.

20. Gross, T.M.; Mastrototaro, J.J. Efficacy and reliability of the continuous glucose monitoring system. Diabetes Technol. Ther. 2000, 2, S19-S26.

21. Hermanides, J.; Vriesendorp, T.M.; Bosman, R.J.; Zandstra, D.F.; Hoekstra, J.B.; Devries, J.H. Glucose variability is associated with intensive care unit mortality. Crit. Care Med. 2010, 38, 838-842.

22. Matthews, D.R.; Hosker, J.P.; Rudenski, A.S.; Naylor, B.A.; Treacher, D.F.; Turner, R.C. Homeostasis model assessment: Insulin resistance and $\beta$-cell function from fasting plasma glucose and insulin concentrations in man. Diabetologia 1985, 28, 412-419. 
23. Madhu, S.V.; Muduli, S.K.; Avasthi, R. Abnormal glycemic profiles by CGMS in obese first-degree relatives of type 2 diabetes mellitus patients. Diabetes Technol. Ther. 2013, 15, 461-465.

24. Borg, R.; Kuenen, J.C.; Carstensen, B.; Zheng, H.; Nathan, D.M.; Heine, R.J.; Nerup, J.; Borch-Johnsen, K.; Witte, D.R.; Group, A.S. Real-life glycaemic profiles in non-diabetic individuals with low fasting glucose and normal HbA1c: The A1C-Derived Average Glucose (ADAG) study. Diabetologia 2010, 53, 1608-1611.

25. Hanefeld, M.; Sulk, S.; Helbig, M.; Thomas, A.; Kohler, C. Differences in Glycemic Variability Between Normoglycemic and Prediabetic Subjects. J. Diabetes Sci. Technol. 2014, 8, 286-290.

26. Fysekidis, M.; Cosson, E.; Banu, I.; Duteil, R.; Cyrille, C.; Valensi, P. Increased glycemic variability and decrease of the postprandial glucose contribution to HbA1c in obese subjects across the glycemic continuum from normal glycemia to first time diagnosed diabetes. Metabolism 2014, 63, 1553-1561.

27. Monnier, L.; Colette, C.; Dunseath, G.J.; Owens, D.R. The loss of postprandial glycemic control precedes stepwise deterioration of fasting with worsening diabetes. Diabetes Care 2007, 30, 263-269.

28. The DECODE Study Group on behalf of the European Diabetes Epidemiology Group. Glucose tolerance and mortality: Comparison of WHO and American Diabetic Association diagnostic criteria. Lancet 1999, 354, 617-621.

29. Pyorala, K. Relationship of glucose tolerance and plasma insulin to the incidence of coronary heart disease: Results from two population studies in Finland. Diabetes Care 1979, 2, 131-141.

30. Després, J.-P.; Lamarche, B.; Mauriège, P.; Cantin, B.; Dagenais, G.R.; Moorjani, S.; Lupien, P.-J. Hyperinsulinemia as an Independent Risk Factor for Ischemic Heart Disease. New Engl. J. Med. 1996, 334, 952-958.

31. Ceriello, A. Point: Postprandial glucose levels are a clinically important treatment target. Diabetes Care 2010, 33, 1905-1907.

32. Chinese Diabetes Society. Chinese clinical guideline for continuous glucose monitoring (2012). Chin. Med. J. (Engl.) 2012, 125, 4167-4174.

33. Ma, C.M.; Yin, F.Z.; Wang, R.; Qin, C.M.; Liu, B.; Lou, D.H.; Lu, Q. Glycemic variability in abdominally obese men with normal glucose tolerance as assessed by continuous glucose monitoring system. Obesity (Silver Spring) 2011, 19, 1616-1622.

34. Sajilata, M.G.; Singhal, R.S.; Kulkarni, P.R. Resistant starch-A review. Compr. Rev. Food Sci. Food Saf. 2006, 5, 1-17.

35. Tappenden, K.A.; McBurney, M.I. Systemic short-chain fatty acids rapidly alter gastrointestinal structure, function, and expression of early response genes. Dig. Dis. Sci. 1998, 43, 1526-1536.

36. Robertson, M.D.; Wright, J.W.; Loizon, E.; Debard, C.; Vidal, H.; Shojaee-Moradie, F.; Russell-Jones, D.; Umpleby, A.M. Insulin-sensitizing effects on muscle and adipose tissue after dietary fiber intake in men and women with metabolic syndrome. J. Clin. Endocrinol. Metab. 2012, 97, 3326-3332.

(C) 2015 by the authors; licensee MDPI, Basel, Switzerland. This article is an open access article distributed under the terms and conditions of the Creative Commons Attribution license (http://creativecommons.org/licenses/by/4.0/). 